\begin{tabular}{|c|l|}
\hline Title & Selective multimode excitation using volume holographic mode multiplexer \\
\hline Author(s) & A oki, Kohei; Okamoto, A tsushi; W akay ama, Y uta; Tomita, A kihisa; Honma, Satoshi \\
\hline Citation & $\begin{array}{l}\text { Optics Letters, 38(5), 769-771 } \\
\text { https://doi.org/10.1364/0L.38.000769 }\end{array}$ \\
\hline Issue Date & 2013-03-01 \\
\hline Doc URL & http://hdl.handle.net/2115/52649 \\
\hline Rights & ○ 2013 Optical Society of A merica, Inc. \\
\hline Type & article \\
\hline File Information & ol-38-5-769.pdf \\
\hline
\end{tabular}

Instructions for use 


\title{
Selective multimode excitation using volume holographic mode multiplexer
}

\author{
Kohei Aoki, ${ }^{1, *}$ Atsushi Okamoto, ${ }^{1}$ Yuta Wakayama, ${ }^{1}$ Akihisa Tomita, ${ }^{1}$ and Satoshi Honma ${ }^{2}$ \\ ${ }^{1}$ Graduate School of Information Science and Technology, Hokkaido University, North-14 West-9, Kita-ku, Sapporo, Hokkaido 060-0814, Japan \\ ${ }^{2}$ Interdisciplinary Graduate School of Medicine and Engineering, University of Yamanashi, 4-3-11 Takeda, Kofu 400-8511, Japan \\ *Corresponding author: aoki@optnet.ist.hokudai.ac.jp
}

Received October 1, 2012; revised February 4, 2013; accepted February 4, 2013; posted February 4, 2013 (Doc. ID 176939); published February 28, 2013

\begin{abstract}
We propose a mode multiplexer based on volume holograms to realize a simple and efficient mode-divisionmultiplexed transmission system that supports a large number of modes. Selective multiexcitation of three spatial modes into a conventional multimode fiber is experimentally demonstrated. This device could potentially multiplex 10 or more modes. Future perspectives of the mode multiplexer for application in mode-division multiplexing are also discussed. (C) 2013 Optical Society of America

OCIS codes: $\quad 060.2330,060.4230,090.4220,090.7330$.
\end{abstract}

As optical transmission systems over single-mode fibers are about to reach their capacity limits [1], new fiberoptics technologies such as space-division multiplexing over multicore fibers [2] and mode-division multiplexing (MDM) [3] have been explored and rapidly developed. In the perspective of cost and energy consumption, MDM is considered one of the most attractive technologies to meet future capacity demands. Recent experiments have demonstrated MDM transmission over few-mode fibers that support two or three spatial modes in combination with multiple-input multiple-output digital signal processing [3-7]. These works were mainly aimed at low-loss and long-distance MDM transmission, which is one of the most important performance indicators in the application of MDM to long-haul systems, and $1200 \mathrm{~km}$ transmission has been achieved in [6].

Another key factor is the number of modes that can be supported by the system. In [8], five-mode ( $\mathrm{LP}_{01}$ and two degenerate $\mathrm{LP}_{11}$ and $\mathrm{LP}_{21}$ modes) transmission was demonstrated, and challenges to attain a larger number of modes were discussed. For mode multiplexers, device simplicity and efficiency are the main issues associated with increasing the number of modes. Most proposed mode multiplexers become complex and lossy as the number of modes increases because one or more additional optical elements, such as beam splitters (BSs), are required in the addition of one mode. In our previous work, to show the possibility of selective multimode excitation that uses only one element, we demonstrated multiple and variable two-mode excitations using a single spatial light modulator (SLM) [9]. The two modes were simultaneously generated by dividing the SLM display into two regions, and these modes were multiplexed at the end of the multimode fiber input. However, the insertion loss was large $(20 \mathrm{~dB})$ because of the low efficiency of the SLM and the nonvertical incidence into the fiber.

In this Letter, we propose a simpler and more efficient mode multiplexer based on volume holograms. The operating principle and the advantages of the volume holographic mode multiplexer are explained, and the experimental results show successful demonstration of a selective three-mode excitation into a conventional graded-index multimode fiber (GI-MMF) with a core diameter of $62.5 \mu \mathrm{m}$.
Figure $\underline{1}$ shows the concept and the operating principle of the volume holographic mode multiplexer, which is very similar to that of volume holographic data storage with angular multiplexing $[10,11]$. During the recording process, a volume hologram is recorded in a holographic medium by interference between a mode beam and a reference beam. The mode beam is transformed into a desired spatial mode, which has intensity and phase fields at the SLM. The mode is changed when the recording has been completed and is ready for the next recording. The reference beam is a plane wave, and its incident angle $\theta$ is shifted corresponding to the change in the spatial mode for angular multiplexed recording. In this process, the spatial modes are recorded in multiple, and the volume holographic mode multiplexer is structured. During the transmission process, reference beams are replaced by signal beams. Owing to the high angular selectivity of the volume holograms, a signal beam is diffracted as one of the recorded spatial modes when the signal beam irradiates the holographic medium from the direction of one of the reference beams. Because the complete wave fields are reconstructed by volume holograms, each diffracted signal beam can be considered as an orthogonal mode and excited into optical fibers as an independent transmission channel. As a recording method, angular multiplexing is the best method because this mode multiplexer can multiplex the different signal beams that come from different directions. However, other methods such as

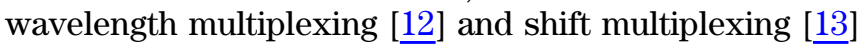

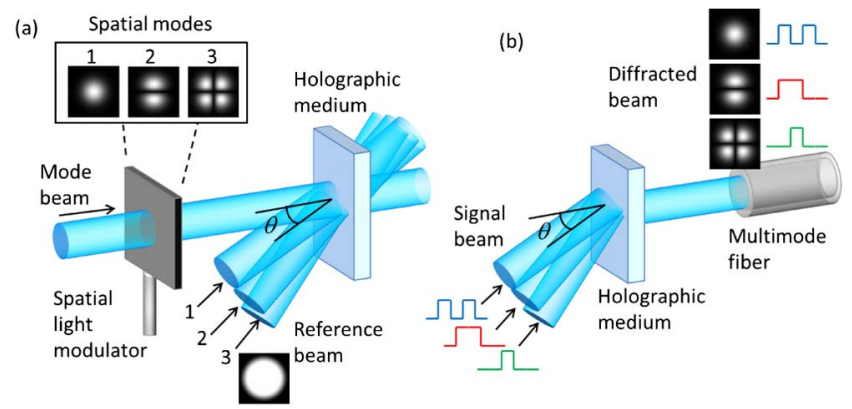

Fig. 1. (Color online) Concept of volume holographic mode multiplexer. (a) Recording process and (b) transmission process. 
can also be employed or combined depending on the situation, e.g., wavelength-division multiplexing (by writing in different wavelengths) or space-division multiplexing (by recording modes at different spots in the holographic medium).

One of the outstanding features of the volume holographic mode multiplexer is its simple structure. This device is simultaneously and selectively able to excite multiple modes using only one holographic medium. Moreover, even if the number of modes increases, no other elements are needed except for the additional reference beams with different incident angles. With respect to efficiency, volume holograms theoretically can achieve $100 \%$ diffraction efficiency for each mode if the holographic medium has a high dynamic range enough for multiple recordings. Alternatively, if the available dynamic range is finite and divided equally among $N$ spatial modes, the diffraction efficiency of each mode would be $1 / N$ for $N<300[11,14]$. In the dominant phase plate-based mode multiplexers introduced in [3], the efficiency would be $1 / 2^{N-1}$ for odd numbers or $1 / 2^{N-2}$ for even numbers because of the BSs used for multiplexing modes. Therefore, the volume holographic mode multiplexer is advantageous when the number of spatial modes is greater than 3. Although the number of modes is limited by the diffraction efficiency, this device can possibly multiplex 10 or more modes, which could support the application of MDM, as suggested in [8]. In addition, polarization multiplexing [15] could enable the treatment of the polarization modes in this multiplexer, indicating that the number of modes will be double. With these advantages, the volume holographic mode multiplexer can realize a simple and efficient mode-divisionmultiplexed transmission system that supports a large number of modes and a high transmission capacity.

Figure 2 shows the experimental setup for multiexcitation of the three spatial modes. To generate the spatial modes, an liquid crystal on silicon-based reflection-type SLM (Holoeye LC-R 1080) was operated as a computergenerated hologram [9], where the Laguerre-Gauss mode approximation [16] was used in the calculation of the mode fields. Displayed hologram patterns and calculated mode fields of the intensity and the phase are shown in

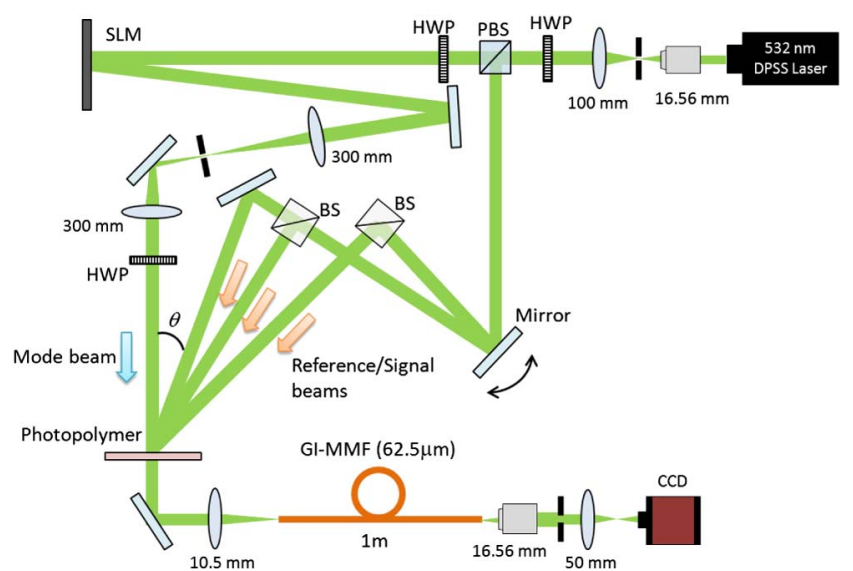

Fig. 2. (Color online) Experimental setup. HWP, half-wave plate; BS, beam splitter, PBS, polarizing beam splitter; SLM, spatial light modulator; GI-MMF, graded-index multimode fiber.

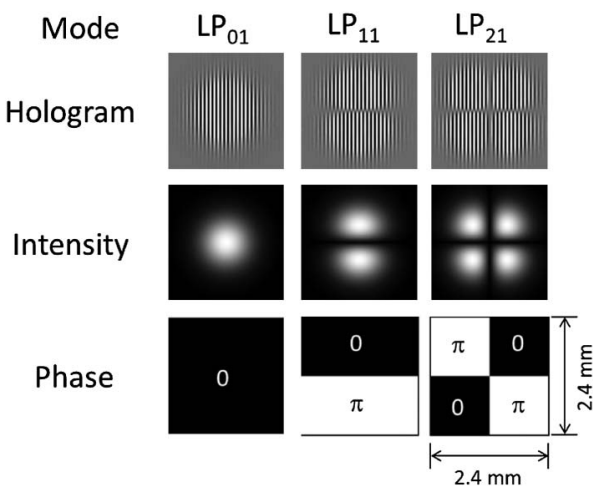

Fig. 3. Displayed hologram patterns on the SLM, and calculated mode fields of the intensity and the phase.

Fig. 3. As a holographic medium, we used the nanogel photopolymer that consists of matrix and acrylic monomers [17], which induces the refractive index change with green light and shows a high diffraction efficiency that reaches $100 \%$ at a thickness of $400 \mu \mathrm{m}$. Therefore, we used a $532 \mathrm{~nm}$ diode pumped solid state laser for the light source. Three modes $\mathrm{LP}_{01}, \mathrm{LP}_{11}$, and $\mathrm{LP}_{21}$ were recorded by angular multiplexing, where the angular differences $\theta$ between the mode and the reference beams were $20^{\circ}$, $30^{\circ}$, and $40^{\circ}$, respectively. Because the measured angular selectivity of the hologram in the photopolymer was $0.1^{\circ}$, the crosstalk, caused by the interference among the multiplexed holograms, was negligible in this experiment. The incident angle of the reference/signal beam was shifted using BSs and a mirror; the BSs can be replaced by mirrors because they were only used for angular shifting. Moreover, they are not needed in practical MDM signal transmission because different lasers would be used for the different signal beams. The recording power values of the mode and the reference beams were approximately 0.6 and $1.5 \mathrm{~mW}$, respectively, and the recording time for each mode was $30 \mathrm{~s}$. Both beams were set to $s$ polarization. The signal beams $(1.5 \mathrm{~mW}$ continuous waves) were diffracted as reconstructed modes, and they were conducted by an aspheric condenser lens $(f=10.5 \mathrm{~mm})$ to a GI-MMF with a core diameter of $62.5 \mu \mathrm{m}$ and a length of $1 \mathrm{~m}$ (Thorlabs GIF625). We used a short fiber to confirm that mode excitations occurred. The output beam from the fiber passed a spatial filter with a circular aperture diameter of $2 \mathrm{~mm}$ to filter out the weak higher-order modes that were converted from the input mode in the fiber, and the beam was observed by a CCD camera. An imaging lens $(f=50 \mathrm{~mm})$ was used to adjust the dimensions of the output to that of the input. The intensity profiles of the three modes at the fiber input and output are shown in Fig. 4. The fiber input modes, which correspond to the holographic reconstructed modes by the mode multiplexer, accurately replicated the original modes (Fig. 3), and the correlation factors between the intensity profiles of the original modes and the reconstructed modes reached 0.95 for each mode. Moreover, the fiber output modes revealed that the three-mode excitation was successfully performed using the volume holographic mode multiplexer, although the output modes were rotated and distorted because of the fiber bending and imperfection of the fiber 


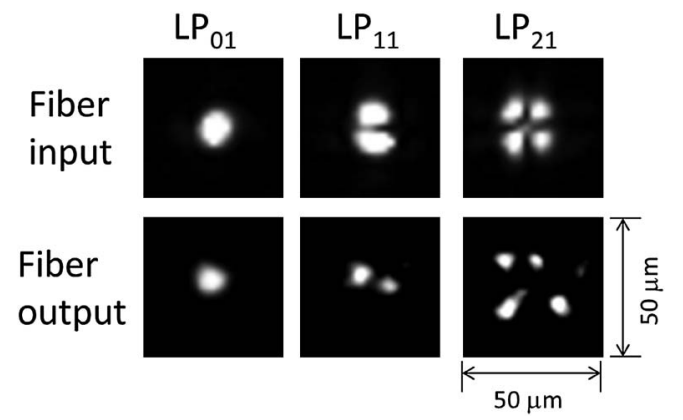

Fig. 4. Intensity profiles of the three modes at the fiber input and output.

end surface. The diffraction efficiency at the photopolymer for each mode was $10 \%$, which means that the device loss was $10 \mathrm{~dB}$. By precisely scheduling the recording, the loss can be improved to $5 \mathrm{~dB}$ if the maximum diffraction efficiency of the photopolymer is achieved.

Because this experiment was conducted at $532 \mathrm{~nm}$, application of the volume holographic mode multiplexer to the communication wavelength should be discussed. The simplest way is to use holographic media that react at the communication wavelength. An infrared-sensitive photorefractive polymer has been reported [18]; however, the diffraction efficiency must be improved ( $40 \%$ at $1550 \mathrm{~nm}$ ). On the other hand, other holographic materials such as photorefractive crystals may be available. Another possible idea would be the dual-wavelength method [19], which diffracts the signal beams with wavelengths that are different from those of the recorded beams by adjusting the incident angles of the signal beams according to Bragg's law. The drawback of this method would be the decrease in diffraction efficiency due to the unavoidable Bragg mismatch caused by the multiplexed holograms. The efficiency decreases as the wavelength difference increases; therefore, it is desirable to employ this technique in cases where the wavelength difference is small. The combination of the dualwavelength method with infrared-sensitive materials or short distance transmission over short-wavelength infrared light could also be considered.

In conclusion, we have proposed a volume holographic mode multiplexer and demonstrated selective multiexcitation of three spatial modes over a conventional multimode fiber with a diameter of $62.5 \mu \mathrm{m}$ and a length of $1 \mathrm{~m}$. This device is advantageous for a large number of modes because of its simple structure. The development of holographic media and the techniques of combining multiple recordings and multiplexed signal transmission (such as the wavelength, space, and polarization) could contribute to the realization of simple, efficient, and high-capacity MDM transmission using this multiplexer. Naturally, optimally designed fibers, amplifiers, and demultiplexers that can support a large number of modes would be required. Further studies are needed to assess the availability of the volume holographic mode multiplexer.

\section{References}

1. R.-J. Essiambre and R. W. Tkach, Proc. IEEE 100, 1035 (2012).

2. J. Sakaguchi, B. J. Puttnam, W. Klaus, Y. Awaji, N. Wada, A. Kanno, T. Kawanishi, K. Imamura, H. Inaba, K. Mukasa, R. Sugizaki, T. Kobayashi, and M. Watanabe, in Optical Fiber Communication Conference 2012 (IEEE, 2012), paper PDP5C.1.

3. S. Randel, R. Ryf, A. Sierra, P. J. Winzer, A. H. Gnauck, C. A. Bolle, R.-J. Essiambre, D. W. Peckham, A. McCurdy, and R. Lingle, Jr., Opt. Express 19, 16697 (2011).

4. C. Koebele, M. Salsi, D. Sperti, P. Tran, P. Brindel, H. Mardoyan, S. Bigo, A. Boutin, F. Verluise, P. Sillard, M. Astruc, L. Provost, F. Cerou, and G. Charlet, Opt. Express 19, 16593 (2011).

5. E. Ip, N. Bai, Y.-K. Huang, E. Mateo, F. Yaman, M.-J. Li, S. Bickham, S. Ten, J. Linares, C. Montero, V. Moreno, X. Prieto, V. Tse, K. M. Chung, A. Lau, H.-Y. Tam, C. Lu, Y. Luo, G.-D. Peng, and G. Li, in European Conference on Optical Communication 2011 (Optical Society of America, 2011), paper PD Th.13.C.2.

6. S. Randel, R. Ryf, A. H. Gnauck, M. A. Mestre, C. Schmidt, R.-J. Essiambre, P. J. Winzer, R. Delbue, P. Pupalaikis, A. Sureka, Y. Sun, X. Jiang, and R. Lingle, Jr., in Optical Fiber Communication Conference 2012 (IEEE, 2012), paper PDP5C.5.

7. R. Ryf, M. A. Mestre, A. H. Gnauck, S. Randel, C. Schmidt, R.-J. Essiambre, P. J. Winzer, R. Delbue, P. Pupalaikis, A. Sureka, Y. Sun, X. Jiang, D. W. Peckham, A. McCurdy, and R. Lingle, Jr., in Optical Fiber Communication Conference 2012 (IEEE, 2012), paper PDP5B.5.

8. M. Salsi, C. Koebele, G. Charlet, and S. Bigo, in Optical Fiber Communication Conference 2012 (IEEE, 2012), paper OTu2C.5.

9. A. Okamoto, K. Aoki, Y. Wakayama, D. Soma, and T. Oda, in Optical Fiber Communication Conference 2012, OSA Technical Digest (Optical Society of America, 2012), paper JW2A-38.

10. P. Hariharan, Optical Holography (Cambridge University, 1996).

11. K. Rastani, Appl. Opt. 32, 3772 (1993).

12. G. A. Rakuljic, V. Leyva, and A. Yariv, Opt. Lett. 17, 1471 (1992).

13. D. Psaltis, M. Levene, A. Pu, G. Barbastathis, and K. Curtis, Opt. Lett. 20, 782 (1995).

14. E. S. Maniloff and K. M. Johnson, J. Appl. Phys. 70, 4702 (1991)

15. W. Su, C. Chen, and Y. Ouyang, Appl. Opt. 46, 3233 (2007).

16. S. Berdague and P. Facq, Appl. Opt. 21, 1950 (1982).

17. J. Ikeda, R. Arai, N. Morishita, C. Katahira, Y. Takatani, S. Yumoto, K. Yokouchi, Y. Hayashi, Y. Tanaka, K. Watanabe, P. B. Lim, and M. Inoue, in Proceedings of International Workshop on Holographic Memories and Display 2009 (University of Tokyo, 2009), paper 3A-4.

18. S. Tay, J. Thomas, M. Eralp, G. Li, R. A. Norwood, A. Schulzgen, M. Yamamoto, S. Barlow, G. A. Walker, S. R. Marder, and N. Peyghambarian, Appl. Phys. Lett. 87, 171105 (2005).

19. E. Chuang and D. Psaltis, Appl. Opt. 36, 8445 (1997). 